\title{
latrogenic pericallosal artery aneurysm after endovascular procedure
}

\author{
Paweł Brzegowy ${ }^{1 A, B, D, E}$, Katarzyna Ciuk²E,F Bartłomiej Łasocha ${ }^{3 A, B}$, Osit Chukwu ${ }^{2 E, F}$, Borys Kwinta ${ }^{4 D}$, \\ Andrzej Urbanik ${ }^{10, E}$, Tadeusz J. Popiela ${ }^{10, E}$ \\ 'Chair of Radiology, Jagiellonian University Medical College, Krakow, Poland \\ ${ }^{2}$ Students'Scientific Group, Chair of Radiology, Jagiellonian University Medical College, Krakow, Poland \\ ${ }^{3}$ Department of Radiology, University Hospital in Krakow, Poland \\ ${ }^{4}$ Department of Neurosurgery and Neurotraumatology, Jagiellonian University Medical College, Krakow, Poland
}

\section{Abstract}

Iatrogenic brain aneurysms are rare and are usually a result of direct trauma to the arterial wall during various neurosurgical or endovascular procedures. Single cases of pericallosal posttraumatic and postsurgical aneurysms were previously reported. Herein, we report the first confirmed case of iatrogenic pericallosal artery aneurysm caused by an arterial wall injury during the endovascular treatment of another aneurysm.

A 50-year-old woman with ruptured anterior communicating artery aneurysm was initially treated with balloon-assisted coiling. During the procedure the tip of a microwire inserted into balloon catheter placed in the pericallosal artery caused a local injury of the inner layer of the vessel wall and vasoconstriction, without bleeding, dissection, or flow disturbances visible in digital subtraction angiography (DSA). Control examination revealed dissecting pericallosal aneurysm. After standard dual-antiplatelet oral preparation, stent-assisted coiling of the pericallosal artery aneurysm was performed with residual contrast filling of the base of the aneurysm sac in control angiography (RROC III). After 6 months the control DSA examination showed entirely cured pericallosal aneurysm (RROC I) and reconstruction of the parent artery.

Successful endovascular treatment of an iatrogenic pericallosal aneurysm was previously reported, and this method seems to be the first-choice treatment. In our case, endovascular stent-assisted coiling also allowed for safe exclusion from circulation of pericallosal dissecting aneurysm, and the implanted stent caused reconstruction of the parent artery, restoring the normal lumen diameter. The second endovascular treatment option considered was implantation of a flow-diverted stent into the pericallosal artery.

Key words: stent-assisted coiling, iatrogenic cerebral aneurysm, pericallosal artery aneurysm.

\section{Introduction}

Intracranial aneurysms are estimated to occur in $3 \%$ or even up to $5-10 \%$ of the global population [1]. Traumatic intracranial aneurysms are uncommon and constitute less than $1 \%$ of all cases. Pseudoaneurysm after penetrating head injuries are more often located peripherally. Iatrogenic aneurysms are usually a result of direct trauma to the arterial wall during various neurosurgical procedures for tumours, aneurysms, and trauma of the skull base and most often involve the internal carotid artery $[2,3]$. The real incidence of these lesions is uncertain and the discovery of them may depend on the timing of performed angiographic examinations [4].

Single cases of pericallosal posttraumatic and postsurgical aneurysms were previously reported $[5,6]$. Herein, we report the first confirmed case of an iatrogenic pericallosal artery aneurysm (periAA) caused by an arterial

Correspondence address:

Dr. Paweł Brzegowy, Chair of Radiology, Jagiellonian University Medical College, 19 Kopernika St., 31-501 Krakow, Poland, phone: +48124001509 ,

e-mail: pawelbrzegowy007@gmail.com

Authors' contribution:

A Study design · B Data collection · C Statistical analysis · D Data interpretation - E Manuscript preparation · F Literature search · G Funds collection 


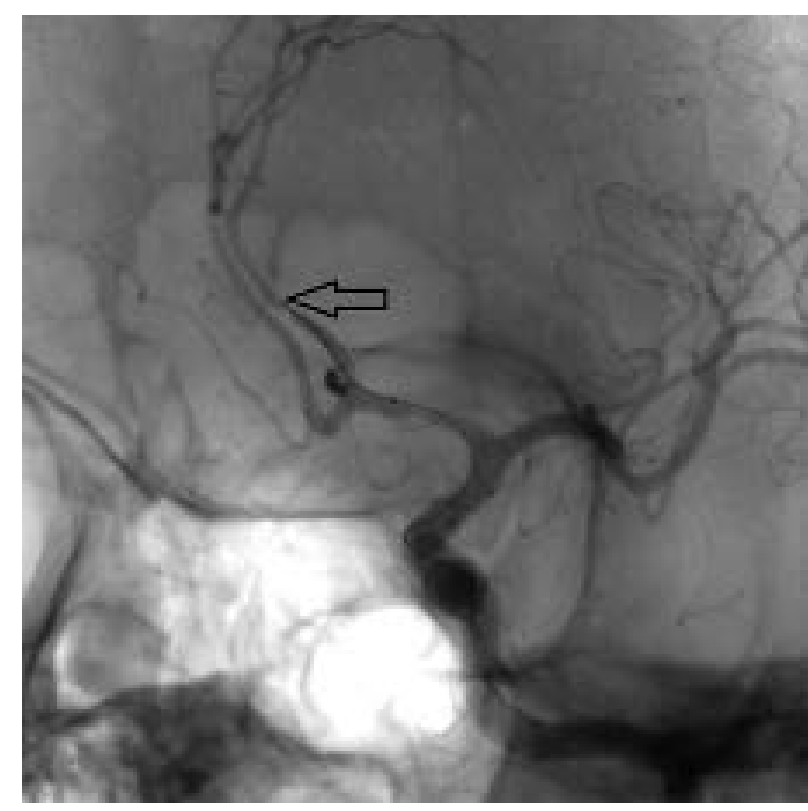

Figure 1. Balloon-assisted coiling of the anterior communicating artery aneurysm. The arrow indicates pericallosal artery injury

wall injury during the endovascular treatment of another aneurysm. A dissecting unruptured aneurysm that was detected in control examination was successfully treated with stent-assisted coiling.

The treatment strategies of iatrogenic aneurysm include both surgical and endovascular procedures and should be based on the patient's clinical data [5]. Because traumatic aneurysms are fragile, prone to rupture, and surrounded by arachnoid scarring, endovascular treatment seems to be the primary option.

\section{Case presentation}

\section{Initial treatment}

A 50-year-old woman with subarachnoid haemorrhage ( 4 in the FISHER Scale) was admitted to the Department

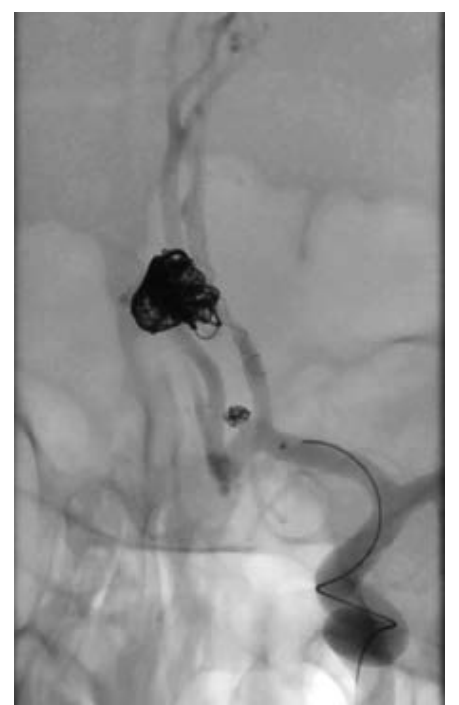

Figure 3. Stent-assisted coiling of pericallosal artery aneurysm

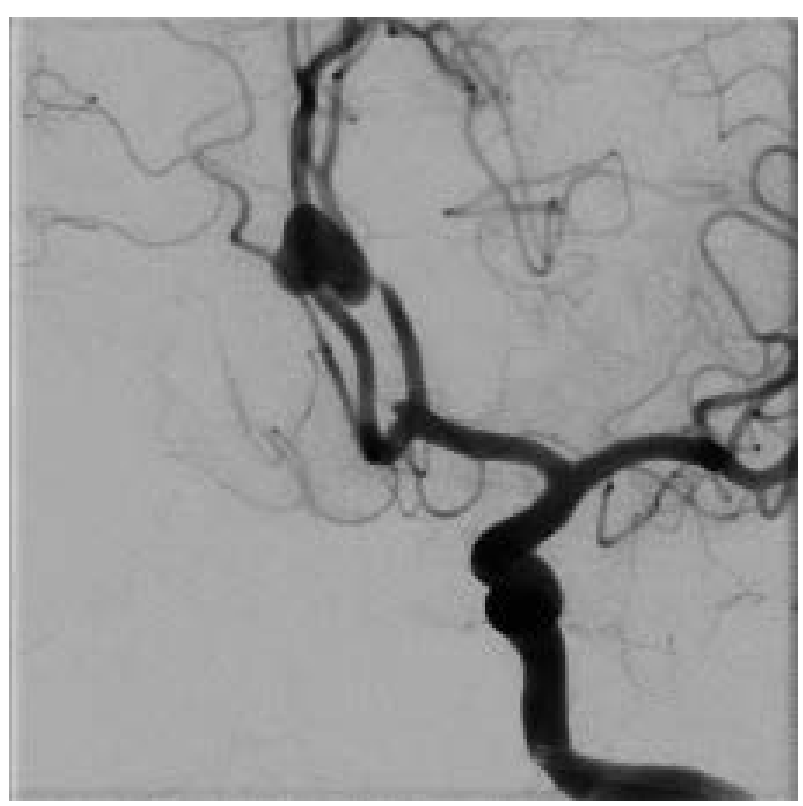

Figure 2. Dissecting pericallosal artery aneurysm in digital subtraction angiography

of Neurosurgery. Computed tomography angiography confirmed the presence of a small $(3 \times 1.5 \mathrm{~mm})$ anterior communicating artery aneurysm (ACoA). The patient was qualified for endovascular treatment. Balloon-assisted coiling of the ACoA was performed under general anaesthesia. Digital subtraction angiography (DSA), performed after the procedure, showed the contrast opacification within the neck of the aneurysm, which equals class II of Raymond-Roy Occlusion Classification (RROC). During the procedure the tip of a microwire (Traxces 14, Microvention Terumo Inc.) inserted into the balloon catheter (Scepter XC, Microvention Terumo Inc) placed in the left pericallosal artery caused local irritation of the inner layer of the vessel wall and vasoconstriction over a length of $1.5 \mathrm{~mm}$, without bleeding, dissection, or flow disturbances visible in DSA (Figure 1).

\section{Follow-up after 1 year}

The control DSA examination performed 12 months after the endovascular procedure of the ACoA revealed a dissecting saccular aneurysm, irregular in shape, about $12 \times$ $6 \mathrm{~mm}$ in diameter, in the place of the previous vasoconstriction of the left pericallosal artery in the A2 segment (Figure 2). The occlusion of coiled ACoA was similar as after the procedure.

\section{The treatment}

After 5 days of standard dual antiplatelet oral preparation (ASA $150 \mathrm{mg}$, clopidogrel $75 \mathrm{mg}$ ) stent-assisted coiling of the periAA was performed. DSA showed 3-mm-long stenosis of the vessel lumen at the level of the aneurysmal neck (Figure 3). Using the jailing technique a Leo baby stent $2.5 \times 25 \mathrm{~mm}$ was implanted into the A2 segment of 
the pericallosal artery, and the aneurysm was embolised with platinum coils. Control angiography showed residual filling of the base of aneurysmal sac (RROC III). Occlusion of the previously treated ACoA remained the same. The patient had no neurological impairments, and the procedure appeared to be without complications.

\section{Follow-up after 6 months}

After 6 months the patient underwent a control DSA examination, which showed entirely cured pericallosal artery aneurysm (RROC I) and improvement of the expansion of the parent vessel. Anterior communicating artery aneurysm remained stable, with inconsiderable residual neck (RROC II) (Figure 4).

\section{Discussion}

Technical development of endovascular neurointerventions allows treatment of a greater number and anatomically more complicated aneurysms using additional balloon catheters, stents, and other instruments. The growing number of devices inserted at the same time inside the artery lumen in a single procedure increases the risk of complications such as perforation, dissection, or thrombus formation.

Until this case there had not been any documented iatrogenic cerebral aneurysm after endovascular procedure. Most reported aneurysms appeared after different neurosurgical vascular and nonvascular procedures [2,3]

Successful endovascular treatment of iatrogenic pericallosal aneurysm was previously reported, and this method seems to be the first-choice treatment [5-7]. In our case, endovascular stent-assisted coiling allowed safe exclusion of pericallosal dissecting aneurysm from circulation, and the implanted stent enabled reconstruction of

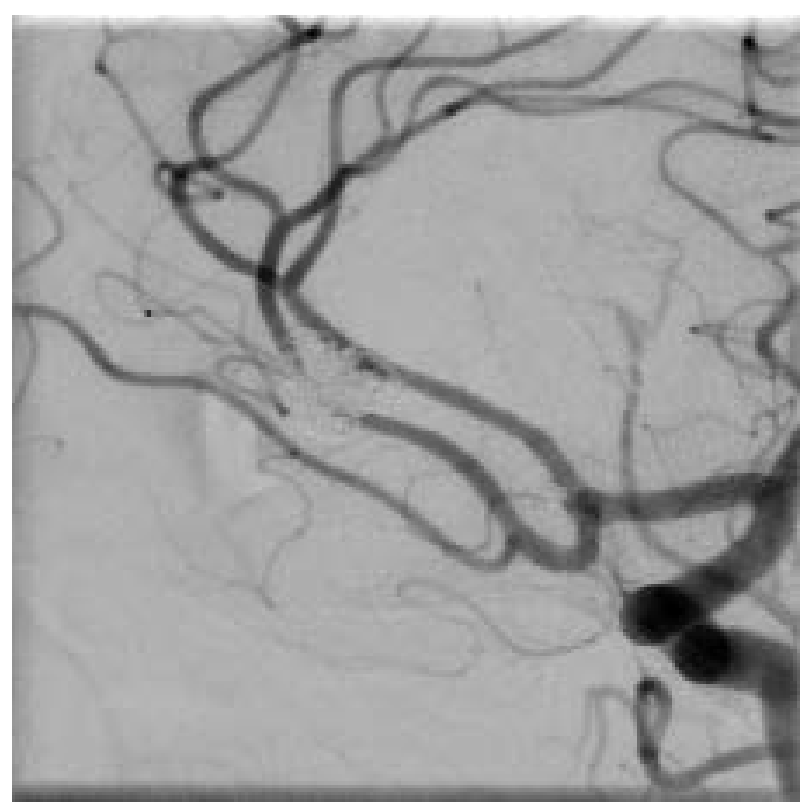

Figure 4. Six-month follow-up digital subtraction angiography - completely occluded pericallosal artery aneurysms. Stable anterior communicating artery aneurysm

the parent artery, restoring the normal lumen diameter. The second considered endovascular treatment option was implantation of a flow-diverted stent into the pericallosal artery [8,9]. However, we found this method to be riskier, causing more potential complications due to the need to use greater force to deliver a larger microcatheter and flow diverter stent into the pericallosal artery with coexisting artery stenosis and not completely cured ACoA aneurysm.

\section{Conflict of interest}

The authors report no conflict of interest.

\section{References}

1. Vlak MH, Algra A, Brandenburg R, et al. Prevalence of unruptured intracranial aneurysms, with emphasis on sex, age, comorbidity, country, and time period: a systematic review and meta-analysis. Lancet Neurol 2011; 10: 626-636.

2. Ciceri EF, Regna-Gladin C, Erbetta A, et al. Iatrogenic intracranial pseudoaneurysms: neuroradiological and therapeutical considerations, including endovascular options. Neurol Sci 2006; 27: 317-322.

3. Aarabi B. Management of traumatic aneurysms caused by high-velocity missile head wounds. Neurosurgery Clinics of North America 1995; 6: 775-797.

4. Benoit BG, Wortzman G. Traumatic cerebral aneurysms. Clinical features and natural history. J Neurol Neurosurg Psychiatry 1973; 36: 127-138.

5. Cohen JE, Rajz G, Itshayek E, et al. Endovascular management of traumatic and iatrogenic aneurysms of the pericallosal artery. Report of two cases. J Neurosurg 2005; 102: 555-557.
6. Van Rooij WJ, Van Rooij SB. Endovascular treatment of traumatic pericallosal artery aneurysms. A case report. Interv Neuroradiol 2013; 19: 56-59.

7. Martines F, Blundo C, Chiappetta F. Surgical treatment of the distal anterior cerebral artery aneurysms. J Neurosurg Sci 1996; 40: 189194.

8. Cagnazzo F, Cappucci M, Dargazanli C, et al. Treatment of distal anterior cerebral artery aneurysms with flow-diverter stents: a single-center experience. AJNR Am J Neuroradiol 2018; 39: 11001106.

9. Cagnazzo F, Perrini P, Dargazanli C, et al. Treatment of unruptured distal anterior circulation aneurysms with flow-diverter stents: a meta-analysis. AJNR Am J Neuroradiol 2019; 40: 687-693. 